\title{
Influence of Wire-Based Impedance-Matching on Helical Antenna Radiation and Limitations of Equivalent Model
}

\author{
Slobodan V. Savić, Member, IEEE, Milan M. Ilić, Senior Member, IEEE, and Antonije R. Djordjević
}

\begin{abstract}
We investigate the influence of a wire-based impedance-matching network for an axial-mode helical antenna on the radiation pattern. This network, proposed in our previous work, comprises a single wire attached to the helix. We show that the matching wire, mounted on a tubular dielectric support and attached to the helix close to the reflector, does not degrade the antenna radiation pattern. We also investigate limitations of the equivalent thin-wire model proposed in our previous work.
\end{abstract}

Keywords - Helical antenna, impedance-matching, radiation pattern, thin-wire model.

\section{INTRODUCTION}

$\mathrm{W}$ HEN a high-gain circularly polarized antenna is required, an axial-mode helical antenna is one of the first choices [1-4]. Although this antenna has been investigated for more than five decades, for most commonly used designs it is poorly matched to the standard $50 \Omega$ transmission lines.

However, a helical antenna can be relatively easily matched [4-7]. In [8] we proposed an impedance-matching network for a helical antenna that comprises a single wire, which has a specific shape. In addition, we formulated an equivalent thin-wire model of the complete helix with the matching wire mounted on a tubular dielectric support, and analyzed the performance of the matching wire and the equivalent model by examining the antenna input reflection coefficient [8]. In the present work, we extend the analysis from [9] to investigate the influence of the matching wire

Paper received March 23, 2019; accepted June 26, 2019. Date of publication July 31,2019 . The associate editor coordinating the review of this manuscript and approving it for publication was Prof. Branko Kolundžija.

This paper is revised and expanded version of the paper presented at the 26th Telecommunications Forum TELFOR 2018 [9].

This work was supported by the Serbian Ministry of Education, Science, and Technological Development under Grant TR32005.

S. V. Savić is with the School of Electrical Engineering, University of Belgrade, Serbia (phone: +381 113218351 ; e-mail: ssavic@etf.bg.ac.rs).

M. M. Ilić is with the School of Electrical Engineering, University of Belgrade, Serbia (phone: +381113218329; e-mail: milanilic@etf.bg.ac.rs) and with the Department of Electrical and Computer Engineering, Colorado State University, Fort Collins, CO 80523-1373, USA.

A. R. Djordjević is with the School of Electrical Engineering, University of Belgrade, Serbia (phone: +381 113370 101; e-mail: edjordja@etf.bg.ac.rs) and with the Serbian Academy of Sciences and Arts. on the radiation pattern of an axial-mode helical antenna. Moreover, we examine how accurately the equivalent model formulated in [8] predicts the antenna far-field behavior. Additionally, we critically examine the limitations of the equivalent thin-wire model of a helical antenna with the matching wire, and through an illustrative example we show that there are situations for which this equivalent model is not appropriate.

\section{Matched HelicAl AnTENNA AND Its EQuivalent MODEL}

Consider a helical antenna made from a copper wire of radius $r=0.525 \mathrm{~mm}$, wound around a hollow PVC tube (carrier) with outer radius $R_{\mathrm{PVCl}}=80 \mathrm{~mm}$ and inner radius $R_{\mathrm{PVC} 2}=76 \mathrm{~mm}$, as shown in Fig. 1(a). The dielectric parameters of the PVC tube are estimated to be $\varepsilon_{\mathrm{r}}=2.8$ and $\tan \delta=0.0049$, and the conductivity of copper is $\sigma_{\mathrm{Cu}}=58 \mathrm{MS} / \mathrm{m}$. The helical antenna has $N=5$ uniform turns with the pitch length $H=120 \mathrm{~mm}$. For the Cartesian coordinate system shown in Fig. 1(a), the helix starts at $z=0$ and ends at $z=N H=600 \mathrm{~mm}$. On both ends of the helix, the PVC tube is extended by $7.5 \mathrm{~mm}$ and a circular copper reflector of a radius $R_{\mathrm{REF}}=200 \mathrm{~mm}$ is placed at the bottom end of the PVC tube. The antenna is fed at its circumference by an SMA coaxial connector. The outer conductor of the SMA connector is connected to the copper reflector and the inner conductor of the SMA connector is connected to the beginning of the helix. The radius of the inner conductor of the SMA connector is $r_{\mathrm{SMA}}=0.635 \mathrm{~mm}$ and its height is $h_{\mathrm{SMA}}=5.25 \mathrm{~mm}$, as shown in Fig. 1(b). The matching-wire bottom end is connected to the inner conductor of the SMA connector and its top end is connected to the first turn of the helical antenna by a vertical, short, straight wire, as shown in Fig. 1(a) and (b).

The helical antenna is matched by a single copper wire connected to the first turn of the helix, as shown in Fig. 1(a) and (b). This matching network (matching wire), proposed in [8], is made from the same copper wire as the helical antenna. This enables formulation of a computationally efficient and accurate equivalent model of the matched helical antenna, as demonstrated in [8]. As it will be shown in this work, when the matching wire is mounted downwards, it practically does not affect the antenna radiation pattern. Thus, it is possible to tune (and optimize) the antenna radiation pattern and reflection coefficient 
completely independently: first, the radiation pattern of the helical antenna without the matching wire is optimized, and thereafter the matching wire is included and tuned (optimized) for the desired reflection coefficient.

For the Cartesian coordinate system shown in Fig. 1(a), the matching-wire axis is parameterized as [8]:

$$
\begin{aligned}
& x=\left(R_{\mathrm{PVC} 1}+r\right) \cos \left(\frac{\pi}{2} t\right), y=\left(R_{\mathrm{PVC} 1}+r\right) \sin \left(\frac{\pi}{2} t\right), \\
& z=z_{\min }+\frac{z_{\text {max }}-z_{\min }}{10^{p_{\mathrm{p}}}-1}\left(10^{p_{\mathrm{p}}\left(\frac{t}{t_{\text {max }}}\right)}-1\right), 0 \leq t \leq t_{\max },
\end{aligned}
$$

where $t$ is an independent parameter $\left(0 \leq t \leq t_{\max }\right)$. In (1) the parameter $t_{\max }$ determines the portion of the helix first turn occupied by the matching wire (e.g., for $t_{\max }=1$, the matching wire extends along one quarter of the first helix turn). The parameter $z_{\text {min }}$ determines the $z$-coordinate of the bottom end of the matching wire, and the parameter $z_{\max }$ determines the $z$-coordinate of the top end of the matching wire. Finally, the parameter $p_{\mathrm{p}}$ determines the curvature of the matching wire - the higher the parameter $p_{\mathrm{p}}$, the closer to the copper reflector the matching wire is bent. As an illustration, the planar projection of the matching wire axis for $p_{\mathrm{p}} \in\{1,2,4\}$ is shown in Fig. 1(c).

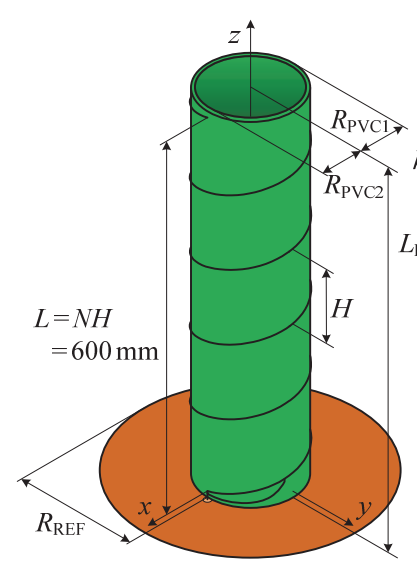

(a)

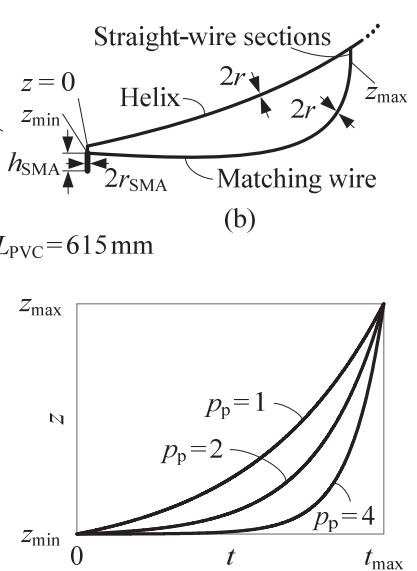

(c)
Fig. 1 (a) A helical antenna with a matching wire. (b) A sketch of the matching wire, whose geometry is governed by (1). (c) A family of curves $z(t)$ defined by (1) for the three specific values of $p_{\mathrm{p}}$.

The two most popular methods for solving steady-state electromagnetic (EM) problems (e.g., antennas and scatterers in the frequency domain) are the finite element method (FEM) [10] and the method of moments (MoM) [11]. Each method, with its advantages and disadvantages, is generally able to model an arbitrary EM problem. On the other hand, due to limitations in computational resources (CPU speed and available memory), an increased complexity of the model can lead to extremely long simulation times. Alternatively, it may not be possible to execute the model on commonly available personal computers (PCs). One way to overcome these limitations is to form an efficient and accurate equivalent model. Instead of considering all details and phenomena explicitly in the original (complete) model, in an equivalent model we make simplifications, such that the equivalent model requires less computational resources while maintaining high accuracy.

Generally, each equivalent model has its own domain of validity. Based on assumptions introduced when forming the equivalent model, its domain of validity could be defined by the range of parameters for which starting assumptions are met and for which it gives results of satisfactory accuracy. On the other hand, if these assumptions are not met, the results of the equivalent model are unpredictable and usually inaccurate. Hence, in order to use an equivalent model properly, we must always keep in mind its limitations, as it will be discussed in Section IV.

For the equivalent model of the matched helical antenna, proposed in [8] and proved to be very efficient and accurate when modeling the antenna reflection coefficient, we will examine here its accuracy and efficiency when modeling the antenna radiation pattern.

Starting from the theory presented in [12] and in the manner described in [8], we consider two-wire transmission lines (a) with and (b) without a PVC substrate, as depicted in Fig. 2. The two-wire transmission line with the PVC substrate will be called "original", and the one without the PVC substrate will be called "equivalent". The conductors of the original two-wire transmission line are made from the same copper wires as the helix and the matching wire, and they lay on a $t_{\mathrm{s}}=R_{\mathrm{PVC} 1}-R_{\mathrm{PVC} 2}=4 \mathrm{~mm}$ thick $\mathrm{PVC}$ substrate, equally thick as the wall of the PVC tube carrying the helical antenna. The width of the substrate is set to $w=10 t_{\mathrm{s}}=40 \mathrm{~mm}$, and the distance between the conductors of the two-wire transmission line is set to $d=10 w=400 \mathrm{~mm}[12]$. Through the quasi-static analysis $[13,14]$ we evaluate the per-unit-length (PUL) capacitance of the original transmission line to be $C^{\prime}=4.98 \mathrm{pF} / \mathrm{m}$ and the inductance to be $L^{\prime}=2.75 \mu \mathrm{H} / \mathrm{m}$.

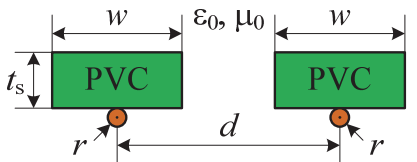

(a)

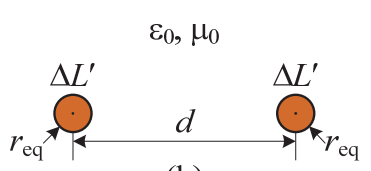

(b)
Fig. 2 Cross-sections of (a) the original and (b) the equivalent two-wire transmission lines.

In order to be mutually equivalent, the transmission lines from Fig. 2(a) and (b) should have the same distance between the conductors $(d)$, the same PUL capacitance, and the same PUL inductance. The equivalent line does not have the PVC substrate. Hence we increase its wire radius to $r_{\mathrm{eq}}=1.5 \mathrm{~mm}$, in order to increase its PUL capacitance, and equalize it with the PUL capacitance of the original line. This slightly decreases the PUL inductance of the equivalent line to $L_{\mathrm{eq}}^{\prime}=2.34 \mu \mathrm{H} / \mathrm{m}$. Finally, in order to equalize the PUL inductance of the original and the equivalent two-wire transmission lines, we add a series PUL inductance $\Delta L^{\prime}=0.205 \mu \mathrm{H} / \mathrm{m}\left[\Delta L^{\prime}=\left(L^{\prime}-L_{\text {eq }}^{\prime}\right) / 2\right]$ into the equivalent line. Adding the series PUL inductance into a wire is a standard feature in all modern full-wave EM simulators [15-17] and it practically does not increase the 
model complexity and the simulation time. The parameters of the equivalent two-wire transmission line ( $r_{\text {eq }}$ and $\left.\Delta L^{\prime}\right)$ will be used when constructing the equivalent wire model of the matched helical antenna, as it will be shown in the next section. The removal of the PVC substrate from the equivalent two-wire transmission line model leads to the removal of the PVC tube in the equivalent wire model of the helical antenna.

As it will be shown in the next section, when the equivalent model of the matched helical antenna is coupled with the thin-wire approximation [18], it leads to a drastic decrease in the model complexity (number of unknowns), which ultimately leads to dramatic reduction in the simulation time. On the other hand, when the equivalent thin-wire model is not used properly, it may lead to inaccurate analysis results.

It is important to note that in the thin-wire approximation it is assumed that: (i) wires are electrically thin (their diameter is much smaller than the wavelength in the surrounding medium), (ii) the radius of the wire curvature is much larger than the wire radius, and (iii) surfaces of two wire segments do not intersect [18]. In order to provide a highly accurate analysis, these assumptions should be met when forming the equivalent model of a matched helical antenna.

\section{NUMERICAL RESULTS}

We construct the equivalent wire model of the matched helical antenna in the WIPL-D Pro software [16]. The WIPL-D Pro solver is based on the MoM, and it uses the thin-wire approximation [18] for straight wire segments. Excluding the copper reflector and the point-generator, this model consists exclusively of equivalent wires (of radius $r_{\text {eq }}$ and with the added PUL inductance $\Delta L^{\prime}$ ) situated in a free space, without the PVC tube. It requires 2103 unknowns and the simulation time is $4 \mathrm{~s}$ per frequency point (when simulated on a modest PC). Using this model, we tune the matching-wire geometric parameters, so that the helical antenna is best matched (with respect to a $50 \Omega$ nominal impedance) in the vicinity of $600 \mathrm{MHz}$ [8]. In (1) we preset $z_{\min }=-2.25 \mathrm{~mm}$ and $z_{\max }=24.41 \mathrm{~mm}$, and tune the other parameters to $t_{\max }=0.8804$ and $p_{\mathrm{p}}=2.609$

. These parameters will be used in all other numerical models in the rest of this paper. For this model, the absolutegain pattern [19] (in two main planes) at $600 \mathrm{MHz}$ is shown in Fig. 3, where it is denoted by "equivalent wire".

Next, we construct the complete model of the matched helical antenna in the ANSYS HFSS software [17]. This software is based on the full-wave FEM. In this model the influence of the PVC tube is included exactly (not through the equivalence). As this model does not contain any equivalences or approximations (apart from numerical discretization, common to all numerical techniques), when fully converged, it can be considered as the reference model. It requires 420929 unknowns and the simulation time is $8 \mathrm{~min} 14 \mathrm{~s}$ per frequency point when executed on the same PC. (Only the final adaptive pass is considered.) As it can be seen from Fig. 3, the results for the "equivalent wire"
WIPL-D model and for the "complete" HFSS model are in an excellent agreement. Thus, the equivalent wire WIPL-D model leads to a significant reduction in simulation time, without any noticeable loss of accuracy in the radiation pattern, as well as in the reflection coefficient [8]. Note that the complete full-wave MoM model with the explicit presence of the PVC tube, assembled in the WIPL-D Pro software, requires 34713 unknowns and the simulation time is $31 \mathrm{~min} 58 \mathrm{~s}$ per frequency point when executed on the same PC. For simplicity, these results are not shown.

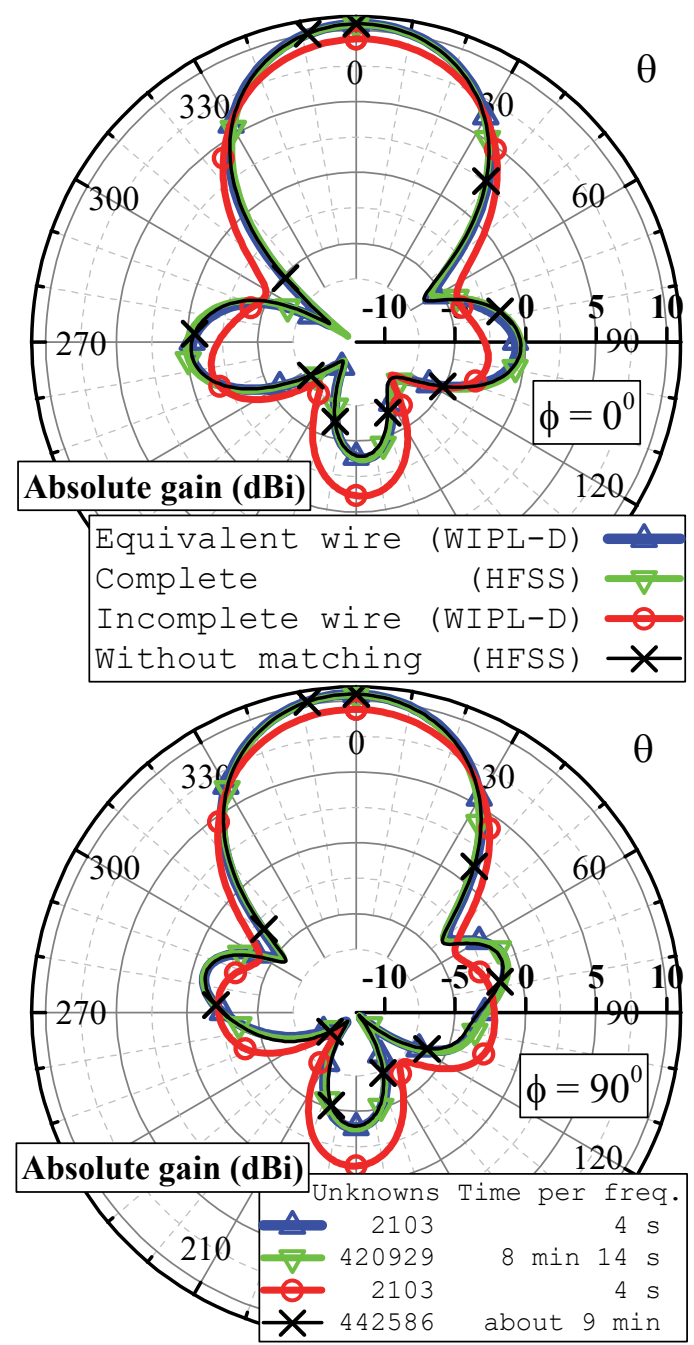

Fig. 3 The absolute gain of the helical antenna with and without the matching wire plotted as a function of zenith angle $\theta$ : comparison of numerical results in two planes $\left(\phi=0^{\circ}\right.$ and $\phi=90^{\circ}, \phi$ is azimuth angle).

In order to determine the influence of the PVC tube on the radiation pattern of the matched helical antenna, we construct an incomplete wire WIPL-D model. We start from the equivalent wire WIPL-D model (with no PVC tube), reset the wire radius to $r$, and remove the PUL inductance $\Delta L^{\prime}$. By doing this, we essentially neglect the influence of the PVC tube on the characteristics of the antenna. This model, as expected, requires the same number of unknowns and the same simulation time as the equivalent wire WIPLD model (2103 unknowns and $4 \mathrm{~s}$ per frequency point, respectively). These results are denoted by "incomplete wire" in Fig. 3. As it can be seen from Fig. 3, these results significantly deviate from other results. Hence we conclude 
that in order to accurately calculate the radiation pattern of the matched helical antenna, the PVC tube must not be omitted from the model. Moreover, if an efficient model is needed, with a small number of unknowns and a short simulation time, the equivalent wire WIPL-D model is an excellent choice.

In order to determine the influence of the matching wire on the radiation pattern of the matched helical antenna, we construct the so-called "without matching" HFSS model. We start from the complete HFSS model and simply remove the matching wire. This model, as expected, requires closely the same number of unknowns and closely the same simulation time as the complete HFSS model. These results are denoted by "without matching" in Fig. 3. As it can be seen from Fig. 3, the matching wire practically does not influence the radiation pattern.

We additionally analyze the influence of the matching wire on the antenna radiation pattern. Namely, by employing the superposition in software [15], we compare the fields radiated by the currents on the matching wire with those radiated by the currents of the helix. This comparison reveals that the radiation intensity of the helix is by $16 \mathrm{~dB}$ higher than that of the matching wire in the direction of the main lobe.

Based on these results, we conclude that when designing a helical antenna with a matching wire at its bottom, the absolute gain and the reflection coefficient can be optimized (or tuned) independently: first, the absolute gain of the antenna without the matching wire is optimized and thereafter the matching wire is included into the model and optimized for the desired reflection coefficient. Note that the matching wire significantly influences the reflection coefficient of the helical antenna [8]. Therefore, it significantly influences the realized gain [19].

\section{LIMITATIONS OF THE EQUIVALENT THIN-WIRE MODEL}

In this section we examine one of the limitations of the equivalent thin-wire model of the helical antenna with the matching wire. Based on theoretical considerations and numerical experiments, we show that the presented equivalent thin-wire model of a helical antenna with a matching wire is not appropriate for a dielectric carrier that has a high relative permittivity. Similar conclusions could be obtained for relatively thick dielectric carriers, but these results are not presented here for the purpose of conciseness.

For the original two-wire transmission line shown in Fig. 2(a), through the quasi-static analysis [13, 14], we calculate the PUL capacitance for the relative permittivity of the dielectric carrier ranging from $\varepsilon_{\mathrm{r}}=2$ to $\varepsilon_{\mathrm{r}}=10$. These results are shown in Fig. 4 by the blue line with blue squares. As expected, the PUL capacitance increases with the increase in relative permittivity of the dielectric carrier.

Next, for the equivalent two-wire transmission line shown in Fig. 2(b), we calculate the PUL capacitance for the equivalent wire radius ranging from $r_{\mathrm{eq}}=1 \mathrm{~mm}$ to $r_{\text {eq }}=5 \mathrm{~mm}$. The results obtained analytically, $\frac{1}{c_{0} C_{\mathrm{eq}}^{\prime}}=120 \Omega \cosh ^{-1}\left(\frac{d}{2 r_{\mathrm{eq}}}\right)$ [20] $\left(c_{0}\right.$ being the speed of

light in a free space), and numerically (through the quasistatic analysis $[13,14]$ ), are shown in Fig. 4 by the red line and red triangles, respectively. These two sets of results are in a very good agreement, thus they can be used interchangeably for the presented example.

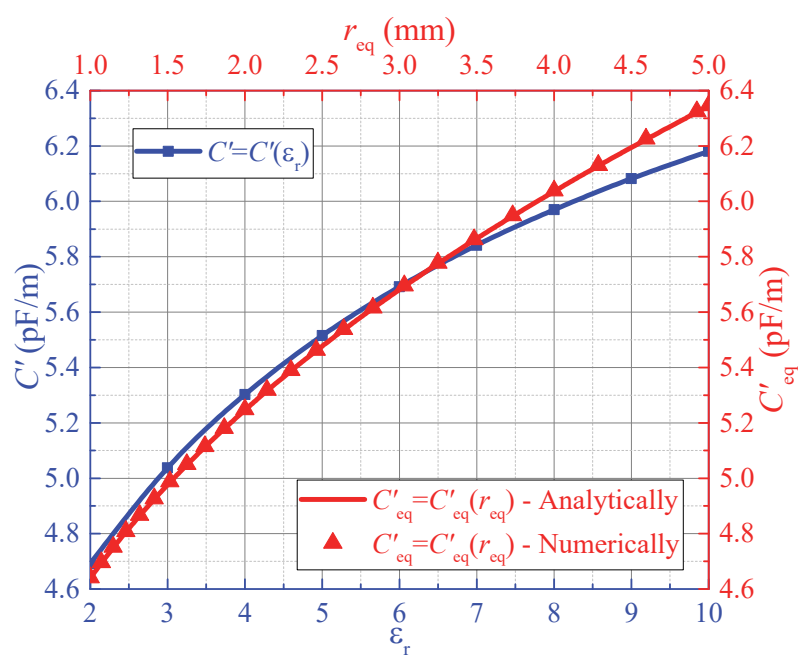

Fig. 4 The PUL capacitance of original two-wire transmission line from Fig. 2(a) vs. relative permittivity of the dielectric carrier (blue line with blue squares), and the PUL capacitance of the equivalent two-wire transmission line from Fig. 2(b) vs. equivalent wire radius (red line and red triangles).

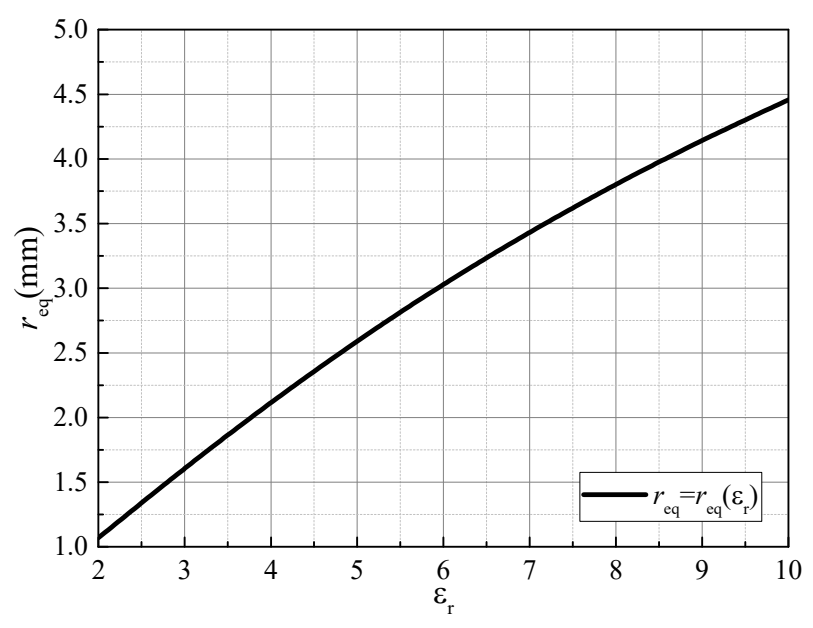

Fig. 5 The equivalent wire radius vs. relative permittivity of the dielectric carrier.

Combining the results from Fig. 4, in Fig. 5 we plot the equivalent wire radius in the equivalent model as a function of the relative permittivity of the dielectric carrier ranging from $\varepsilon_{\mathrm{r}}=2$ to $\varepsilon_{\mathrm{r}}=10$ in the original model. As it is evident from Fig. 5, a dielectric carrier with a higher relative permittivity leads to a larger radius of the equivalent wire in the equivalent model. In this example, the equivalent wire radius grows practically linearly with relative permittivity. For a high enough permittivity of the dielectric carrier, the radius of the equivalent wire can be so large that the assumptions (i) and (iii) from Section II for 
the thin-wire approximation are no longer met, as shown in the next example.

Consider a helical antenna with the dielectric carrier having $\varepsilon_{\mathrm{r}}=10$. The PUL capacitance of the corresponding original two-wire transmission line is $C^{\prime}=6.18 \mathrm{pF} / \mathrm{m}$, and its inductance is $L^{\prime}=2.75 \mu \mathrm{H} / \mathrm{m}$ (the same as in the previous example, since the change in the permittivity of the dielectric support does not affect the PUL inductance of the line). The radius of the equivalent wire is $r_{\text {eq }}=4.45 \mathrm{~mm}$, and the PUL inductance of the equivalent two-wire transmission line is $L_{\text {eq }}^{\prime}=1.9 \mu \mathrm{H} / \mathrm{m}$. In order to establish the equivalence, we add a series PUL inductance $\Delta L^{\prime}=\left(L^{\prime}-L_{\mathrm{eq}}^{\prime}\right) / 2=0.425 \mu \mathrm{H} / \mathrm{m}$ into the equivalent model.

The reflection coefficient obtained by utilizing the equivalent thin-wire model and the complete full-wave FEM model is shown in Fig. 6 for this example $\left(\varepsilon_{\mathrm{r}}=10\right)$. In the same figure, the results from [8] (when the relative permittivity of the dielectric carrier is $\varepsilon_{\mathrm{r}}=2.8$ ) are shown for comparison.

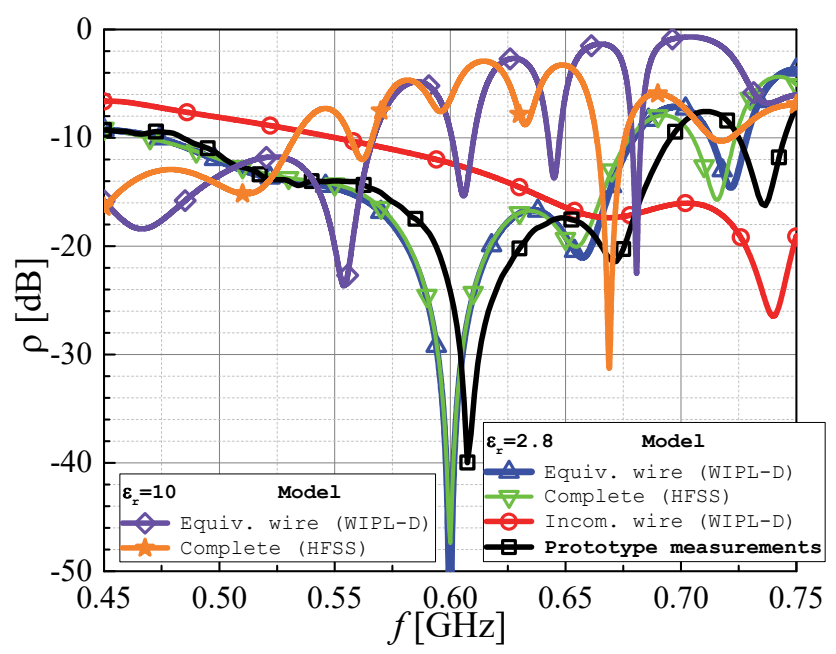

Fig. 6 The reflection coefficient of the helical antenna with the matching wire: comparison of simulation models $\left(\varepsilon_{\mathrm{r}}=2.8\right.$ and $\left.\varepsilon_{\mathrm{r}}=10\right)$ and measurements $\left(\varepsilon_{\mathrm{r}}=2.8\right)$.

As can be seen from Fig. 6 and as discussed in [8], for dielectric carriers whose relative permittivity is not too high (e.g., $\varepsilon_{\mathrm{r}}=2.8$ ) the results obtained by the equivalent thinwire model and by the complete model are in an excellent agreement. Further, both sets of results are in a very good agreement with the prototype measurements. On the other hand, for the dielectric carriers with a higher relative permittivity (e.g., $\varepsilon_{\mathrm{r}}=10$ ), the results obtained by the equivalent thin-wire model and by the complete model do not match. Hence, in this case the equivalent thin-wire model cannot be used for the efficient and accurate design of the helical antenna with the matching wire.

For numerical techniques which use straight wire segments [16], in order to accurately approximate the curvature of the matching wire and the helix, we must use relatively short wire segments. For the equivalent model with a large radius of the equivalent wire, the length to radius ratio of wire segments can be small (wire segments can be stubby), which is challenging for modeling with the thin-wire approximation.

In the equivalent model of the matched helical antenna with a large radius of equivalent wires, some wire segments can significantly penetrate other wire segments, which is additionally challenging for modeling with the thin-wire approximation. A detail of the equivalent thin-wire model of matched helical antenna is shown in Fig. 7. From that figure it can be seen how one stubby wire segment is significantly penetrating other stubby wire segment.

The rapid increase of radius of the equivalent wire with the increase of the permittivity of the dielectric support represents a significant limitation of the equivalent thinwire model of a helical antenna with a matching wire. Thus, its application must be limited to problems where the radius of the equivalent wire is not too large. This limitation can be bypassed using the method from [21]. In that method, a wire, coated with a dielectric layer, is treated by considering currents and charges that are located on the wire surface with an additional layer of charges located at the outer surface of the coating. However, in the available commercial software, only currents and charges located on the wire surface are involved in the thin-wire model. Hence, the application of the method from [21] requires writing a customized code.

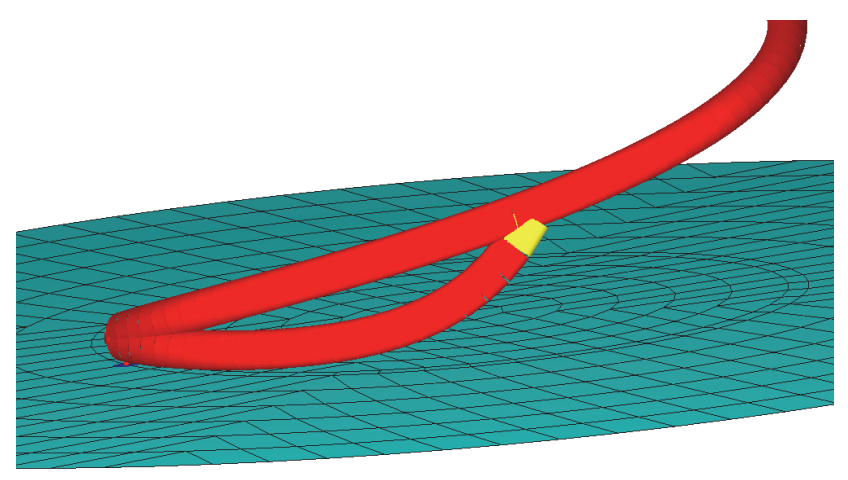

Fig. 7 Detail from the equivalent thin-wire model of a helical antenna with the matching wire. Part of the first turn of the helix with the matching wire is shown; one stubby wire segment which significantly penetrates other stubby wire segment is highlighted in yellow.

\section{CONCLUSION}

We examined the influence of the matching network (matching wire) on the radiation pattern of an axial-mode helical antenna. Based on the presented results, we conclude that the matching wire practically does not influence the radiation pattern and that the reflection coefficient and the radiation pattern of the helical antenna can be optimized (tuned) independently. This significantly simplifies the design process.

We investigated limitations of the equivalent thin-wire model of a helical antenna with the matching wire. Based on results presented here, we conclude that an equivalent thin-wire model of the helical antenna with the matching wire can be used for small to moderate permittivities of dielectric carriers. 


\section{REFERENCES}

[1] G. A. Thiele and W. L. Stutzman, Antenna Theory and Design. New York: J. Wiley, 1981.

[2] J. D. Kraus, "Helical Beam Antennas," Electronics, vol. 20, pp. 109111, April 1947.

[3] J. D. Kraus and J. C. Williamson, "Characteristics of Helical Antennas Radiating in the Axial Mode," J. Appl. Phys., vol. 19, no. 1, pp. 87-96, January 1948.

[4] A. R. Djordjevic, A. G. Zajić, M. M. Ilić, and G. L. Stüber, "Optimization of Helical Antennas [Antenna Designer's Notebook]," IEEE Antennas Propag. Mag., vol. 48, no. 6, pp. 107-115, December 2006.

[5] R. J. Stegen, "Impedance Matching of Helical Antennas," IEEE Trans. Antennas Propag., vol. 12, no. 1, pp. 125-126, January 1964.

[6] J. D. Kraus, "A 50-Ohm Input Impedance for Helical Beam Antennas," IEEE Trans. Antennas Propag., vol. AP-25, no. 6, pp. 913-913, November 1977.

[7] M. M. Ilić, S. V. Savić, and P. Djondović, "Internal Matching of Uhf Helical Antenna Exciters for Magnetic Resonance Imaging," in Proc. of 3rd Int. Conf. on Electrical, Electronic and Computing Engineering (IcETRAN), Zlatibor, Serbia, 2016, pp. API1.1.1-5.

[8] S. V. Savić, M. M. Ilić, and A. R. Djordjević, "Design of Internal Wire-Based Impedance Matching of Helical Antennas Using an Equivalent Thin-Wire Model," International Journal of Antennas and Propagation, vol. 2017, pp. 1-5, December 2017.

[9] S. V. Savić, M. M. Ilić, and A. R. Djordjević, "Influence of WireBased Impedance-Matching on Helical Antenna Radiation," in Proceedings of 26th Telecommunications Forum TELFOR 2018, Belgrade, Serbia, 2018.

[10] J.-M. Jin, The Finite Element Method in Electromagnetics. Hoboken, New Jersey: John Wiley \& Sons, 2014.
[11] R. F. Harrington, Field Computation by Moment Methods (Ieee Series on Electromagnetic Wave Theory). New Jersey Wiley-IEEE Press, 1993.

[12] B. D. Popović and A. Nešić, "Generalisation of the Concept of Equivalent Radius of Thin Cylindrical Antennas," IEE Proc. H Microw., Optics and Antennas, vol. 131, no. 3, pp. 153-158, June 1984.

[13] A. R. Djordjević, M. B. Baždar, R. F. Harrington, and T. K. Sarkar, Linpar for Windows: Matrix Parameters for Multiconductor Transmission Lines, Version 2.0. Norwood, Massachusetts: Artech House, 1999.

[14] ANSYS. Ansys Maxwell 15.0.0. ANSYS, 2014 http://www.ansys.com.

[15] A. R. Djordjević, M. B. Baždar, V. V. Petrović, D. I. Olćan, T. K Sarkar, and R. F. Harrington, Awas for Windows Version 2.0: Analysis of Wire Antennas and Scatterers. Norwood, Massachusetts: Artech House, 2002.

[16] WIPL-D. Wipl-D Pro 11.0. WIPL-D, 2013, http://www.wipld.com.

[17] ANSYS. Ansys Hfss 15.0.0. ANSYS, 2014, http://www.ansys.com.

[18] B. D. Popović, M. B. Dragović, and A. R. Djordjević, Analysis and Synthesis of Wire Antennas (Antennas Series). New York: John Wiley \& Sons - Research Studies Press, 1982.

[19] "IEEE Standard Definitions of Terms for Antennas," IEEE Std 145 1993, pp. 1-36, July 181993.

[20] R. E. Collin, Foundations for Microwave Engineering, 2 ed. (Ieee Press Series on Electromagnetic Wave Theory). New York: WileyIEEE Press, 2001.

[21] B. D. Popović, A. R. Djordjević, and N. M. Kirćanski, "Simple Method for Analysis of Dielectric-Coated Wire Antennas," Radio and Elect. Eng., vol. 51, no. 3, pp. 141-145, March 1981. 